\title{
Panel: Open Source in the Public Sector
}

\author{
Björn Lundell ${ }^{1}$, Morten Amundsen ${ }^{2}$, Rishab Aiyer Ghosh ${ }^{3}$, \\ Jean-Luc Hardy ${ }^{4}$, and Per-Ola Sjöswärd ${ }^{5}$ \\ ${ }^{1}$ University of Skövde, Sweden \\ bjorn. Iundell@his.se \\ ${ }^{2}$ Friprog - The Norwegian Open Source Competence Center, Norway \\ ${ }^{3}$ MERIT, The Netherlands \\ ${ }^{4}$ Eurocontrol, France \\ ${ }^{5}$ Swedish National Police, Sweden
}

Open Source Software (OSS) is becoming mainstream, and it is perhaps not surprising that public sector organisations seek to explore the potential of OSS in financially difficult times. Today, OSS has become an issue of strategic importance for many public sector organisations. In addition, related to OSS, many organisations and governments are also acknowledging Open Standards as important for addressing various lock-in scenarios.

In many countries, there are national policies and governmental initiatives aimed to support the public sector in better utilizing OSS and Open Standards. For example, the Open Source Observatory and Repository, OSOR ${ }^{1}$, is an EU-initiative for exchanging information, experiences and OSS-based software for use in the public sector. In specific countries there are national initiatives, such as "The Netherlands in Open Connection", which is an action plan for OSS and open standards in the public sector that has been adopted in The Netherlands.

Many public sector organisations are currently exploring the potential of Open Source, and for some it has become core to their way of working. Open Source is currently evolving and we have yet to see its full potential. In this panel, the goal is to stimulate discussion on the current trends, opportunities, risks, and experiences to be considered with the wider adoption of OSS (and Open Standards) in public sector organisations. Specifically, the panel seeks to address the following question:

What inhibitors are there to leveraging the advantages of OSS in a Public Sector context, and how might those inhibitors be ameliorated?

Related to the above (general) question, the discussion will address the following issues:

- To what extent (and in what areas) is OSS used in public sector organisations?

- What is the situation, in various countries and public sector organisations, regarding policies for (or against) use of OSS and Open Standards?

- Is there a government-sponsored mandate or preference for OSS and Open Standards in your country?

- How is OSS adopted in public sector organisations and who provides technology support for such software?

\footnotetext{
${ }^{1}$ See http://www.osor.eu/
} 
- What are the most significant inhibitors to adoption and use of OSS in different countries and public sector organisations?

- What inhibitors are there to leveraging the advantages of Open Standards in a Public Sector context, and how might those inhibitors be ameliorated?

- What is the experience for how any barriers to adoption and use of OSS in a public sector organisation might be reduced?

- How can public sector organisations develop healthy ecosystems around OSS and what is their role in such open projects?

- How can (and should) public sector organisations collaborate, at regional, national, and global levels?

The panel will start with short position statements by the panellists and a dialogue between them. Significant time will be allocated to an open discussion on the issues with the audience, and those attending are invited to raise their own concerns and share their own experiences. 